\title{
A Shortcut to Fmoc-Protected Phosphinic Pseudodipeptidic Blocks
}

\author{
Supporting Information
}

\author{
Magdalini Matziari and Athanasios Yiotakis
}

University of Athens, Department of Chemistry, Laboratory of Organic Chemistry, Panepistimiopolis Zografou 15771, Athens, Greece 


\section{TABLE OF CONTENTS:}

General considerations and Abbreviations........................................

General method for the synthesis of compounds of type 2 ' ....................

General method for the synthesis of compounds of type 2 ...................6

General method for the synthesis of compounds of type $3 \ldots \ldots \ldots \ldots \ldots \ldots \ldots . \ldots 7$

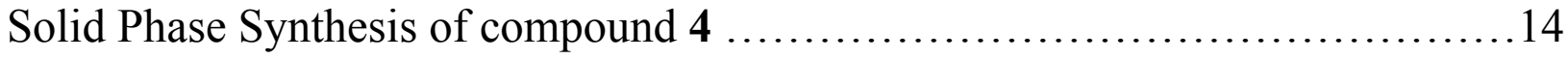


General: All of the compounds, for which analytical and spectroscopic data are quoted, were homogenous by TLC. TLC analyses were performed using silica gel plates (E. Merck silica gel 60 F-254) and components were visualized by the following methods: ultraviolet light absorbance and charring after spraying with a solution of $\left(\mathrm{NH}_{4}\right) \mathrm{HSO}_{4}$. Column chromatography was carried out on silica gel (E.Merck, 70-230 mesh), height $42 \mathrm{~cm}$ and diameter $1.5 \mathrm{~cm}$. HPLC

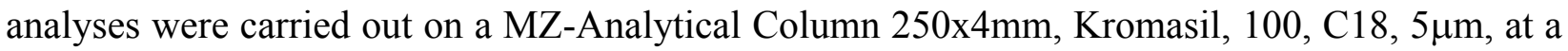
flow rate of $0.5 \mathrm{~mL} / \mathrm{min}$. Solvent A: $10 \% \mathrm{CH}_{3} \mathrm{CN}, 90 \% \mathrm{H}_{2} \mathrm{O}, 0.1 \%$ TFA. Solvent B: $90 \%$ $\mathrm{CH}_{3} \mathrm{CN}, 10 \% \mathrm{H}_{2} \mathrm{O}, 0.09 \%$ TFA. The following gradients were used: $1 . t=0$ min $(0 \% \mathrm{~B}), t=10 \mathrm{~min}$ (25\% B), $t=45 \min (75 \% \mathrm{~B}), t=50 \min (100 \% \mathrm{~B}), t=55 \min (100 \% \mathrm{~B}), t=60 \min (40 \% \mathrm{~B})$, for compounds 3a-3l and 2. $t=0 \min (0 \% \mathrm{~B}), t=5 \min (25 \% \mathrm{~B}), t=50 \mathrm{~min}(25 \% \mathrm{~B}), t=55 \mathrm{~min}(100 \%$ B), $t=60 \min (100 \% \mathrm{~B}), t=65 \min (40 \% \mathrm{~B})$, for compound 4 . Eluted peaks were detected at 254 nm. Given times (where more than one) correspond to two pairs of diastereoisomers and are counted in minutes. All the compounds were characterized by ${ }^{1} \mathrm{H},{ }^{13} \mathrm{C}$ and ${ }^{31} \mathrm{P}-\mathrm{NMR}$ spectroscopy. ${ }^{1} \mathrm{H},{ }^{13} \mathrm{C}$ and ${ }^{31} \mathrm{P}-\mathrm{NMR}$ spectra were recorded on a $200 \mathrm{MHz}$ Mercury Varian spectrometer. ${ }^{13} \mathrm{C}$ and ${ }^{31} \mathrm{P}$-NMR spectra are fully proton decoupled. ${ }^{31} \mathrm{P}$ - chemical shifts are reported on $\delta$ scale (in ppm) downfield from $85 \% \mathrm{H}_{3} \mathrm{PO}_{4}$. ESI mass spectral analysis was performed on a mass sprectrometer MSQ Surveyor, Finnigan at the Laboratory of Organic Chemistry, University of Athens, using direct sample injection. Negative or positive ion ESI spectra were acquired by adjusting the needle and cone voltages accordingly. Commercially available reagents, solvents and starting materials were purchased from Aldrich, Merck, Sigma, Labscan, and Fluka and used without further purification.

Abbreviations: AcOH: acetic acid, AcOEt: ethyl acetate, $\mathrm{Bu}^{\mathrm{t}}$ : tert-butyl, DCM: dichloromethane, DIC: disopropylcarbodiimide, DIPEA: diisopropylethylamine, DMF: dimethylformamide, $\mathrm{EDC} \cdot \mathrm{HCl}$ : 1-ethyl-3-(3'-dimethylaminopropyl)carbodiimide $\mathrm{HCl}, \quad \mathrm{Et}_{2} \mathrm{O}$ : diethyl ether, EtOH: ethanol, HMDS: 1,1,1,3,3,3-hexamethyldisilazane, HOBt: 1hydroxybenzotriazole, $\mathrm{MeOH}$ : methanol, P.E. $40-60^{\circ} \mathrm{C}$ : petroleum ether $40-60^{\circ} \mathrm{C}$, TFA: trifluoroacetic acid, TIS: triisopropylsilane, Z: benzyloxycarbonyl. 


\section{General method for the synthesis of compounds of type 2':}<smiles>[R1]C(C[PH2]([2H])O)C(=O)OCC</smiles>

Ammonium hypophosphate salt $\mathrm{H}_{2} \mathrm{P}(\mathrm{O})\left(\mathrm{O}^{-} \mathrm{NH}_{4}{ }^{+}\right)$is produced by mixing equimolecular quantities of commercially available aqueous solutions of $\mathrm{H}_{3} \mathrm{PO}_{2} 50 \%$ and $\mathrm{NH}_{3} 25 \%$ at $0^{\circ} \mathrm{C}$, evaporation of water, and drying over $\mathrm{P}_{2} \mathrm{O}_{5}$ to constant weight. Acrylic acid esters are either commercially available or synthesised using known procedures.

In a 250-ml-round-bottomed flask, 4 gr $(48.2 \mathrm{mmol})$ of dry $\mathrm{H}_{2} \mathrm{P}(\mathrm{O})\left(\mathrm{O}^{-} \mathrm{NH}_{4}{ }^{+}\right)$are placed and HMDS ( $1 \mathrm{eq}, 10.3 \mathrm{~mL}$ ) is added under an inert atmosphere. After $2 \mathrm{~h}$ at $110^{\circ} \mathrm{C}$, the reaction mixture is cooled to $\mathrm{rt}, 60 \mathrm{~mL}$ of anhydrous DCM are added and after cooling to $0^{\circ} \mathrm{C}$, the acrylic derivative (0.2 eq, $9.6 \mathrm{mmol}$ ) is added neat, or dissolved in DCM, during $1 / 2$ to $1 \mathrm{~h}$. After $24 \mathrm{~h}, 10$ $\mathrm{mL}$ of $\mathrm{EtOH}$ are added, the reaction mixture is evaporated in vacuo, then dissolved in 5\% $\mathrm{NaHCO}_{3}$, extracted with ether, acidified with $\mathrm{HCl} 6 \mathrm{M}$ and extracted with AcOEt. Yields: 75\%$90 \%$.

\section{2-Benzyl-3-hydroxyphosphinoyl-propionic acid ethyl ester (2’a):}<smiles>CCOC(=O)C(Cc1ccccc1)CP(=O)(O)O</smiles>

$$
\begin{gathered}
\mathrm{C}_{12} \mathrm{H}_{17} \mathrm{O}_{4} \mathrm{P} \\
\text { Exact Mass: } 256,09 \\
\text { Mol. Wt.: } 256,23
\end{gathered}
$$

Viscous colourless oil. Yield 89\%, 2.19 g. TLC $R_{f}\left(\mathrm{CHCl}_{3} / \mathrm{MeOH} / \mathrm{AcOH}=7 / 2 / 1\right) 0.59$. ${ }^{1} \mathrm{H}$ NMR $\left(200 \mathrm{MHz}, \mathrm{CDCl}_{3}\right) \delta 1.27(\mathrm{t}, J=7.2 \mathrm{~Hz}, 3 \mathrm{H}), 1.72-2.21(\mathrm{~m}, 2 \mathrm{H}), 2.81-3.18(\mathrm{~m}, 3 \mathrm{H}), 4.05(\mathrm{q}, J=7.2$ $\mathrm{Hz}, 2 \mathrm{H}), 7.05(\mathrm{~d}, J=564 \mathrm{~Hz}, 1 \mathrm{H}), 7.07-7.38(\mathrm{~m}, 5 \mathrm{H}) ;{ }^{13} \mathrm{C}-\mathrm{NMR}\left(50 \mathrm{MHz}, \mathrm{CDCl}_{3}\right) \delta 13.9,30.7$ (d, $\left.{ }^{1} J_{\mathrm{PC}}=98.9 \mathrm{~Hz}\right), 38.2,40.1,61.4,126.5,128.2,129.5,137.9,173.8 ;{ }^{31} \mathrm{P}-\mathrm{NMR}\left(81 \mathrm{MHz}, \mathrm{CDCl}_{3}\right) \delta$ 36.92 . 
2-Hydroxyphosphinoylmethyl-4-methyl-pentanoic acid ethyl ester (2’b):
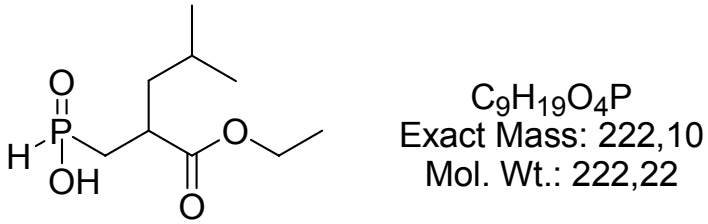

Viscous colourless oil. Yield 82\%, 1.75 g. TLC $R_{f}\left(\mathrm{CHCl}_{3} / \mathrm{MeOH} / \mathrm{AcOH}=7 / 2 / 1\right)$ 0.67. ${ }^{1} \mathrm{H}$ NMR $\left(200 \mathrm{MHz}, \mathrm{CDCl}_{3}\right) \delta 1.05(\mathrm{dd}, J=6.2 \mathrm{~Hz}, 12.1 \mathrm{~Hz}, 6 \mathrm{H}), 1.27$ (t, J=7.1 Hz, 3H), 1.35-1.45 (m, $1 \mathrm{H}), 1.53-2.25(\mathrm{~m}, 4 \mathrm{H}), 2.79-2.86(\mathrm{~m}, 1 \mathrm{H}), 4.17$ (q, $J=7.1 \mathrm{~Hz}, 2 \mathrm{H}), 7.19(\mathrm{~d}, J=561 \mathrm{~Hz}, 1 \mathrm{H}) ;{ }^{13} \mathrm{C}-$ NMR (50 MHz, $\left.\mathrm{CDCl}_{3}\right) \delta 14.1,22.3,22.7,25.7,31.3\left(\mathrm{~d},{ }^{1} J_{\mathrm{PC}}=85.6 \mathrm{~Hz}\right), 36.2,43.8,60.8,175.5$;

${ }^{31} \mathrm{P}-\mathrm{NMR}\left(81 \mathrm{MHz}, \mathrm{CDCl}_{3}\right) \delta 37.23$.

3-Hydroxyphosphinoyl-2-methyl-propionic acid ethyl ester (2'c):

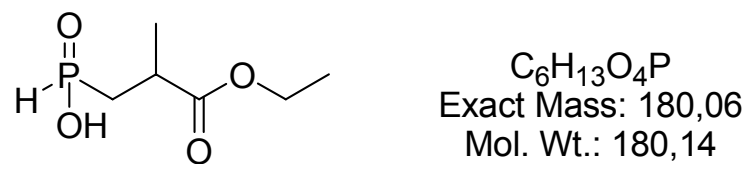

Viscous colourless oil. Yield 79\%, $1.37 \mathrm{~g}$. TLC $R_{f}\left(\mathrm{CHCl}_{3} / \mathrm{MeOH} / \mathrm{AcOH}=7 / 2 / 1\right)$ 0.64. ${ }^{1} \mathrm{H} \mathrm{NMR}$ $\left(200 \mathrm{MHz}, \mathrm{CDCl}_{3}\right) \delta 1.24-1.35(\mathrm{~m}, 6 \mathrm{H}), 1.75-1.94(\mathrm{~m}, 1 \mathrm{H}), 2.09-2.33(\mathrm{~m}, 1 \mathrm{H}), 2.82-2.98(\mathrm{~m}$, $1 \mathrm{H}), 4.17$ (q, $J=7.1 \mathrm{~Hz}, 2 \mathrm{H}), 7.24(\mathrm{~d}, J=565 \mathrm{~Hz}, 1 \mathrm{H}) ;{ }^{13} \mathrm{C}-\mathrm{NMR}\left(50 \mathrm{MHz}, \mathrm{CDCl}_{3}\right) \delta 14.0,19.0$, $31.5\left(\mathrm{~d},{ }^{1} J_{\mathrm{PC}}=91.4 \mathrm{~Hz}\right), 34.4,60.5,175.4 ; \delta^{31} \mathrm{P}-\mathrm{NMR}\left(81 \mathrm{MHz}, \mathrm{CDCl}_{3}\right) \delta 36.91$.

3-Hydroxyphosphinoyl-propionic acid ethyl ester (2'd):<smiles>[2H][PH](=O)CCC(=O)OCC</smiles>

$$
\begin{gathered}
\mathrm{C}_{5} \mathrm{H}_{11} \mathrm{O}_{4} \mathrm{P} \\
\text { Exact Mass: } 166,04 \\
\text { Mol. Wt.: } 166,11
\end{gathered}
$$

Viscous colourless oil. Yield 75\%, 1.20 g. TLC $R_{f}\left(\mathrm{CHCl}_{3} / \mathrm{MeOH} / \mathrm{AcOH}=7 / 2 / 1\right) 0.54$. ${ }^{1} \mathrm{H}$ NMR $\left(200 \mathrm{MHz}, \mathrm{CDCl}_{3}\right) \delta 1.27(\mathrm{t}, J=7.1 \mathrm{~Hz}, 3 \mathrm{H}), 2.01-2.16(\mathrm{~m}, 2 \mathrm{H}), 2.58-2.73(\mathrm{~m}, 2 \mathrm{H}), 4.17(\mathrm{q}, J=7.1$ 
$\mathrm{Hz}, 2 \mathrm{H}), 7.24(\mathrm{~d}, J=563 \mathrm{~Hz}, 1 \mathrm{H}) ;{ }^{13} \mathrm{C}-\mathrm{NMR}\left(50 \mathrm{MHz}, \mathrm{CDCl}_{3}\right) \delta 14.0,22.1\left(\mathrm{~d},{ }^{1} J_{\mathrm{PC}}=92.9 \mathrm{~Hz}\right), 26$. 7, 60.1, $172.2 ;{ }^{31} \mathrm{P}-\mathrm{NMR}\left(81 \mathrm{MHz}, \mathrm{CDCl}_{3}\right) \delta 38.07$.

General method for the synthesis of compounds of type 2: Phosphinic acids of type 2' (1 $\mathrm{mmol})$ are dissolved in $\mathrm{MeOH}(2 \mathrm{~mL})$ and aq. solution of $\mathrm{NaOH} 4 \mathrm{M}(2 \mathrm{~mL})$ is added and stirred at $\mathrm{rt}$ until completion of the reaction, as indicated by TLC. The reaction mixture is concentrated to dryness and $\mathrm{HCl} /$ dioxane $4 \mathrm{M}(2 \mathrm{~mL})$ is added. Filtration of salts and removal of solvent affords the pure products, as oils. Yields: 90\%-98\%.

\section{2-Benzyl-3-hydroxyphosphinoyl-propionic acid (2a):}
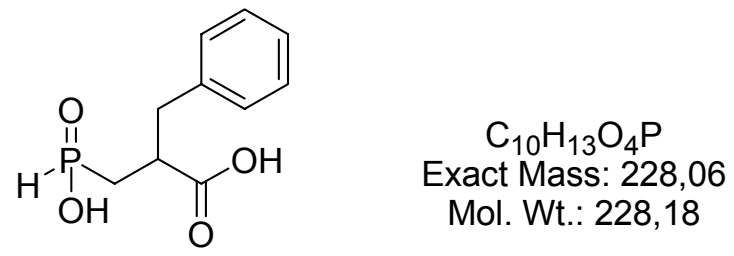

Viscous colourless oil. Yield 98\%, 1.91 g. TLC $R_{f}\left(\mathrm{CHCl}_{3} / \mathrm{MeOH} / \mathrm{AcOH}=7 / 2 / 1\right)$ 0.34. ${ }^{1} \mathrm{H}$ NMR $\left(200 \mathrm{MHz}, \mathrm{CDCl}_{3}\right) \delta 1.73-2.23(\mathrm{~m}, 2 \mathrm{H}), 2.73-2.81(\mathrm{~m}, 1 \mathrm{H}), 3.01-3.22(\mathrm{~m}, 2 \mathrm{H}), 7.07(\mathrm{~d}, J=569$ $\mathrm{Hz}, 1 \mathrm{H}), 7.15-7.31(\mathrm{~m}, 5 \mathrm{H}) ;{ }^{13} \mathrm{C}-\mathrm{NMR}\left(50 \mathrm{MHz}, \mathrm{CDCl}_{3}\right) \delta 30.8(\mathrm{~d}, J=96.4 \mathrm{~Hz}), 38.3,39.4,127.5$, $128.2,128.5,137.1,178.2 ;{ }^{31} \mathrm{P}-\mathrm{NMR}\left(81 \mathrm{MHz}, \mathrm{CDCl}_{3}\right) \delta 36.48$.

\section{2-Hydroxyphosphinoylmethyl-4-methyl-pentanoic acid (2b):}
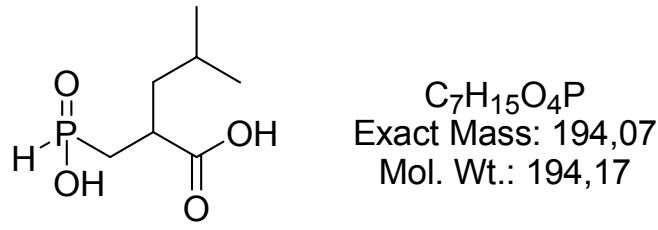

Viscous colourless oil. Yield 97\%, 1.48 g. TLC $R_{f}\left(\mathrm{CHCl}_{3} / \mathrm{MeOH} / \mathrm{AcOH}=7 / 2 / 1\right) 0.47 .{ }^{1} \mathrm{H}$ NMR $\left(200 \mathrm{MHz}, \mathrm{CDCl}_{3}\right) \delta 1.06(\mathrm{dd}, J=6.7 \mathrm{~Hz}, 13.1 \mathrm{~Hz}, 6 \mathrm{H}), 1.34-1.41(\mathrm{~m}, 1 \mathrm{H}), 1.59-1.73(\mathrm{~m}, 2 \mathrm{H})$, 1.83-2.30 (m, 2H) 2.75-2.92 (m, 1H), $7.22(\mathrm{~d}, J=565 \mathrm{~Hz}, 1 \mathrm{H}) ;{ }^{13} \mathrm{C}-\mathrm{NMR}\left(50 \mathrm{MHz}, \mathrm{CDCl}_{3}\right) \delta$ 22.3, 22.7, 25.6, $30.4\left(\mathrm{~d},{ }^{1} J_{\mathrm{PC}}=92.1 \mathrm{~Hz}\right), 34.2,43.1,178.4 ;{ }^{31} \mathrm{P}-\mathrm{NMR}\left(81 \mathrm{MHz}, \mathrm{CDCl}_{3}\right) \delta 36.33$. 


\section{3-Hydroxyphosphinoyl-2-methyl-propionic acid (2c):}<smiles>CC(CP(=O)(O)O)C(=O)O</smiles>

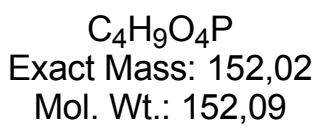

Viscous colourless oil. Yield 95\%, 1.10 g. TLC $R_{f}\left(\mathrm{CHCl}_{3} / \mathrm{MeOH} / \mathrm{AcOH}=7 / 2 / 1\right) 0.23 .{ }^{1} \mathrm{H}$ NMR $\left(200 \mathrm{MHz}, \mathrm{CDCl}_{3}\right) \delta 1.22-1.37(\mathrm{~m}, 3 \mathrm{H}), 1.82-2.08(\mathrm{~m}, 1 \mathrm{H}), 2.10-2.38(\mathrm{~m}, 1 \mathrm{H}), 2.84-3.15(\mathrm{~m}$, $1 \mathrm{H}), 7.23(\mathrm{~d}, J=568 \mathrm{~Hz}, 1 \mathrm{H}) ;{ }^{13} \mathrm{C}-\mathrm{NMR}\left(50 \mathrm{MHz}, \mathrm{CDCl}_{3}\right) \delta 18.8,31.6\left(\mathrm{~d},{ }^{1} J_{\mathrm{PC}}=88.1 \mathrm{~Hz}\right), 34.2$, $178.4 ; \delta^{31} \mathrm{P}-\mathrm{NMR}\left(81 \mathrm{MHz}, \mathrm{CDCl}_{3}\right) \delta 36.78$.

\section{3-Hydroxyphosphinoyl-propionic acid (2d):}<smiles>O=C(O)CCP(=O)(O)O</smiles>

$$
\begin{gathered}
\mathrm{C}_{3} \mathrm{H}_{7} \mathrm{O}_{4} \mathrm{P} \\
\text { Exact Mass: } 138,01 \\
\text { Mol. Wt.: } 138,06
\end{gathered}
$$

Viscous colourless oil. Yield 90\%, $0.90 \mathrm{~g}$. TLC $R_{f}\left(\mathrm{CHCl}_{3} / \mathrm{MeOH} / \mathrm{AcOH}=7 / 2 / 1\right) 0.18 .{ }^{1} \mathrm{H}$ NMR $\left(200 \mathrm{MHz}, \mathrm{CDCl}_{3}\right) \delta 2.06-2.14(\mathrm{~m}, 2 \mathrm{H}), 2.59-2.75(\mathrm{~m}, 2 \mathrm{H}), 7.25(\mathrm{~d}, J=564 \mathrm{~Hz}, 1 \mathrm{H}) ;{ }^{13} \mathrm{C}-\mathrm{NMR}$ $\left(50 \mathrm{MHz}, \mathrm{CDCl}_{3}\right) \delta 24.9\left(\mathrm{~d},{ }^{1} J_{\mathrm{PC}}=89.5 \mathrm{~Hz}\right), 29.3,175.5 ;{ }^{31} \mathrm{P}-\mathrm{NMR}\left(81 \mathrm{MHz}, \mathrm{CDCl}_{3}\right) \delta 38.09$.

General method for the synthesis of compounds of type 3: Phosphinic acid of type 2 (1 mmol) is dissolved in $\mathrm{AcCl} / \mathrm{AcOH}(10 / 2 \mathrm{~mL})$ and $\mathrm{FmocNH}_{2}(1 \mathrm{mmol})$ is added. The appropriate aldehyde $(1.1 \mathrm{mmol})$ is added at $0^{\circ} \mathrm{C}$ and the reaction mixture is allowed to stir at $\mathrm{rt}$ for $6 \mathrm{~h}$. Evaporation of the solvents and purification by column chromatography, using $\mathrm{CHCl}_{3} / \mathrm{MeOH} / \mathrm{AcOH}=9 / 0.8 / 0.5$ as eluent system, affords the pure products as white solids, after trituration with Petroleum Ether $40-60^{\circ} \mathrm{C}$. Yields given below. 
2-Benzyl-3-\{[(9H-fluoren-9-ylmethoxycarbonylamino)-methyl]-hydroxy-phosphinoyl\}propionic acid (3a):

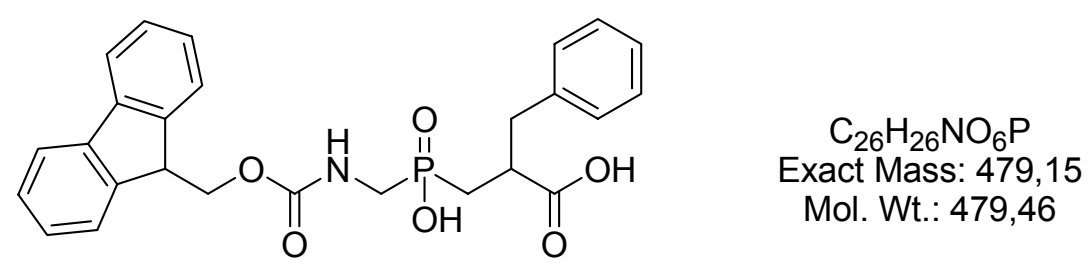

White solid. Yield 56\%, $270 \mathrm{mg}$. TLC $R_{f}\left(\mathrm{CHCl}_{3} / \mathrm{MeOH} / \mathrm{AcOH}=70 / 5 / 5\right) 0.16$. HPLC $\mathrm{t}_{\mathrm{R}} 35.95 .{ }^{1} \mathrm{H}$ NMR (200 MHz, d $\mathrm{d}_{6}$-DMSO) $\delta 1.60-1.82(\mathrm{~m}, 1 \mathrm{H}), 1.85-2.13(\mathrm{~m}, 1 \mathrm{H}), 2.78-3.10(\mathrm{~m}, 3 \mathrm{H}), 3.23-$ $3.39(\mathrm{~m}, 1 \mathrm{H}), 4.09-4.36(\mathrm{~m}, 1 \mathrm{H}), 4.15-4.51(\mathrm{~m}, 3 \mathrm{H}), 7.20-7.91(\mathrm{~m}, 14 \mathrm{H}) ;{ }^{13} \mathrm{C}-\mathrm{NMR}\left(50 \mathrm{MHz}, \mathrm{d}_{6^{-}}\right.$ DMSO) $\delta 29.0\left(\mathrm{~d},{ }^{1} J_{\mathrm{PC}}=89.5 \mathrm{~Hz}\right), 41.5,46.7,65.9,120.2,125.3,126.3,127.1,127.7,128.3$, $129.1,138.9,140.8,143.9,156.3,175.3\left(\mathrm{~d},{ }^{3} \mathrm{~J}_{\mathrm{PC}}=8.5 \mathrm{~Hz}\right) ;{ }^{31} \mathrm{P}-\mathrm{NMR}\left(81 \mathrm{MHz}, \mathrm{d}_{6}\right.$-DMSO) $\delta$ 43.14. ESMS $m / z$ calcd for $\mathrm{C}_{26} \mathrm{H}_{25} \mathrm{NO}_{6} \mathrm{P}(\mathrm{M}-\mathrm{H})^{-} 478.1$, found 478.3 .

\section{2-Benzyl-3-\{[1-(9H-fluoren-9-ylmethoxycarbonylamino)-ethyl]-hydroxy-phosphinoyl\}- propionic acid (3b):}
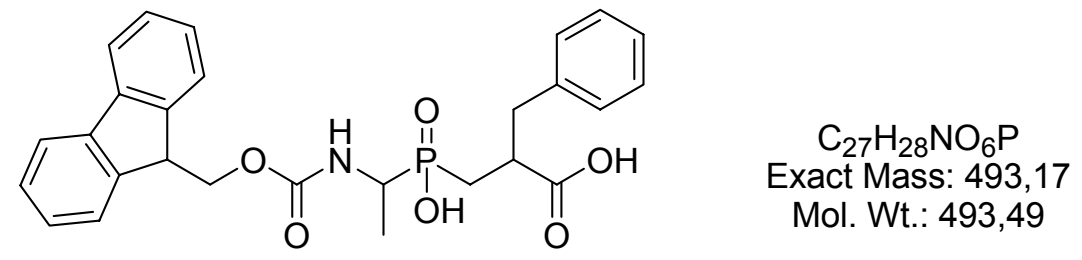

White solid. Yield 57\%, $281 \mathrm{mg}$. TLC $R_{f}\left(\mathrm{CHCl}_{3} / \mathrm{MeOH} / \mathrm{AcOH}=70 / 5 / 5\right) \quad 0.20$. HPLC $\mathrm{t}_{\mathrm{R}}$ 36.85/37.03. ${ }^{1} \mathrm{H}$ NMR (200 MHz, $\mathrm{d}_{6}$-DMSO) $\delta 1.18-1.31$ (m, 3H), 1.62-1.82 (m, 1H), 1.85-2.10 $(\mathrm{m}, 1 \mathrm{H}), 2.69-3.05(\mathrm{~m}, 3 \mathrm{H}), 3.65-3.90(\mathrm{~m}, 1 \mathrm{H}), 4.18-4.39(\mathrm{~m}, 3 \mathrm{H}), 7.18-7.91(\mathrm{~m}, 14 \mathrm{H}) ;{ }^{13} \mathrm{C}-\mathrm{NMR}$ (50 MHz, d 6 -DMSO) $\delta 22.1\left(\mathrm{~d},{ }^{2} J_{\mathrm{PC}}=9.3 \mathrm{~Hz}\right), 27.3\left(\mathrm{~d},{ }^{1} J_{\mathrm{PC}}=86.2 \mathrm{~Hz}\right), 27.7\left(\mathrm{~d},{ }^{1} J_{\mathrm{PC}}=86.2 \mathrm{~Hz}\right)$, $45.6\left(\mathrm{~d},{ }^{1} J_{\mathrm{PC}}=105.9 \mathrm{~Hz}\right), 46.1\left(\mathrm{~d},{ }^{1} J_{\mathrm{PC}}=105.9 \mathrm{~Hz}\right), 46.7,65.9,120.1,125.3,125.4,126.3,127.1$, 127.7, 128.2, 129.0, 129.1, 138.8, 140.8, 143.8, 143.9, $155.9\left(\mathrm{~d},{ }^{3} J_{\mathrm{PC}}=4.9 \mathrm{~Hz}\right), 175.3\left(\mathrm{~d},{ }^{3} J_{\mathrm{PC}}=9.6\right.$ $\mathrm{Hz}$ ); ${ }^{31} \mathrm{P}-\mathrm{NMR}$ (81 MHz, $\mathrm{d}_{6}$-DMSO) $\delta 46.77 / 46.86$. ESMS $m / z$ calcd for $\mathrm{C}_{27} \mathrm{H}_{27} \mathrm{NO}_{6} \mathrm{P}(\mathrm{M}-\mathrm{H})^{-}$ 492.2, found 492.3 . 
2-Benzyl-3-\{[1-(9H-fluoren-9-ylmethoxycarbonylamino)-2-methyl-propyl]-hydroxyphosphinoyl\}-propionic acid (3c):
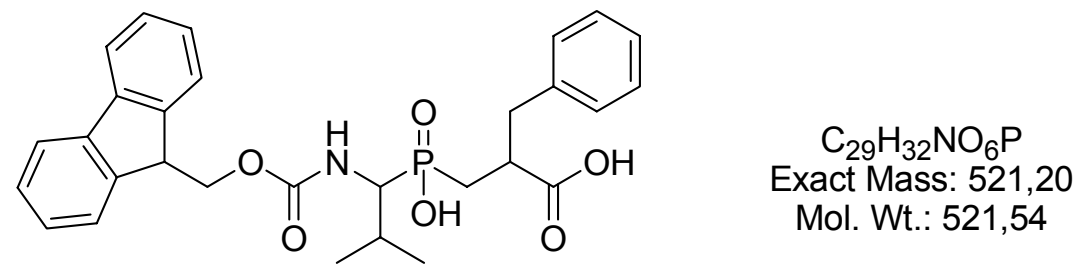

White solid. Yield 55\%, $287 \mathrm{mg}$. TLC $R_{f}\left(\mathrm{CHCl}_{3} / \mathrm{MeOH} / \mathrm{AcOH}=70 / 5 / 5\right)$ 0.26. HPLC $\mathrm{t}_{\mathrm{R}}$ 41.08/41.41. ${ }^{1} \mathrm{H}$ NMR (200 MHz, d 6 -DMSO) $\delta$ 0.82-0.97 (m, 6H), 1.16-2.02 (m, 5H), 2.73-3.05 $(\mathrm{m}, 3 \mathrm{H}), 3.46-3.70,(\mathrm{~m}, 1 \mathrm{H}), 4.09-4.39(\mathrm{~m}, 3 \mathrm{H}), 7.17-7.91(\mathrm{~m}, 14 \mathrm{H})$;

${ }^{13} \mathrm{C}-\mathrm{NMR}\left(50 \mathrm{MHz}, \mathrm{d}_{6}\right.$-DMSO) $\delta 18.7,20.9,22.5,28.5\left(\mathrm{~d},{ }^{1} J_{\mathrm{PC}}=87.1 \mathrm{~Hz}\right), 29.0\left(\mathrm{~d},{ }^{1} J_{\mathrm{PC}}=87.1\right.$ $\mathrm{Hz}), 46.7,55.3\left(\mathrm{~d},{ }^{1} J_{\mathrm{PC}}=105.9 \mathrm{~Hz}\right), 55.8\left(\mathrm{~d},{ }^{1} J_{\mathrm{PC}}=104.5 \mathrm{~Hz}\right), 66.0,120.1,125.4,125.4,126.3$, 127.1, 127.7, 128.2, 129.0, 129.1, 138.8, 140.7, 143.8, 143.9, 156.7 (d, $\left.{ }^{3} J_{\mathrm{PC}}=5.1 \mathrm{~Hz}\right), 175.3(\mathrm{~d}$, ${ }^{3} J_{\mathrm{PC}}=9.5 \mathrm{~Hz}$ ); ${ }^{31} \mathrm{P}-\mathrm{NMR}\left(81 \mathrm{MHz}, \mathrm{d}_{6}\right.$-DMSO) $\delta 46.14 / 46.55$. ESMS $m / z$ calcd for $\mathrm{C}_{29} \mathrm{H}_{31} \mathrm{NO}_{6} \mathrm{P}$ $(\mathrm{M}-\mathrm{H})^{-}$520.2, found 520.3.

\section{2-Benzyl-3-\{[1-(9H-fluoren-9-ylmethoxycarbonylamino)-3-methyl-butyl]-hydroxy-} phosphinoyl\}-propionic acid (3d):
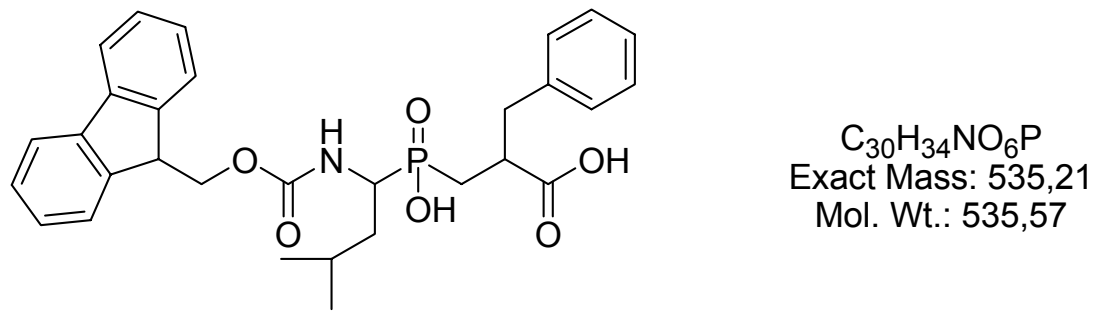

White solid. Yield 62\%, $332 \mathrm{mg}$. TLC $R_{f}\left(\mathrm{CHCl}_{3} / \mathrm{MeOH} / \mathrm{AcOH}=70 / 5 / 5\right)$ 0.30. HPLC $\mathrm{t}_{\mathrm{R}}$ 44.02/44.36. ${ }^{1} \mathrm{H}$ NMR (200 MHz, d ${ }_{6}$-DMSO) $\delta$ 0.81-1.05 (m, 6H), 1.15-2.10 (m, 5H), 2.76-3.10 $(\mathrm{m}, 3 \mathrm{H}), 3.63-3.85,(\mathrm{~m}, 1 \mathrm{H}), 4.18-4.35(\mathrm{~m}, 3 \mathrm{H}), 7.17-7.91(\mathrm{~m}, 14 \mathrm{H}) ;{ }^{13} \mathrm{C}-\mathrm{NMR}\left(50 \mathrm{MHz}, \mathrm{d}_{6^{-}}\right.$ DMSO) $\delta 20.9,23.4,23.9,24.1,28.3\left(\mathrm{~d},{ }^{1} J_{\mathrm{PC}}=88.7 \mathrm{~Hz}\right), 28.7\left(\mathrm{~d},{ }^{1} J_{\mathrm{PC}}=88.7 \mathrm{~Hz}\right), 35.3,46.8,48.5$ $\left(\mathrm{d},{ }^{1} J_{\mathrm{PC}}=107.8 \mathrm{~Hz}\right), 48.9\left(\mathrm{~d},{ }^{1} J_{\mathrm{PC}}=107.8 \mathrm{~Hz}\right), 65.7,120.2,125.2,125.4,126.3,127.1,127.7$, 
128.2, 129.0, 129.1, 138.8, 140.8, 143.7, 144.0, $156.2\left(\mathrm{~d},{ }^{3} J_{\mathrm{PC}}=4.4 \mathrm{~Hz}\right), 175.3\left(\mathrm{~d},{ }^{3} J_{\mathrm{PC}}=11.2 \mathrm{~Hz}\right)$; ${ }^{31} \mathrm{P}-\mathrm{NMR}$ (81 MHz, $\left.\mathrm{d}_{6}-\mathrm{DMSO}\right) \delta 46.95$. ESMS $m / z$ calcd for $\mathrm{C}_{30} \mathrm{H}_{33} \mathrm{NO}_{6} \mathrm{P}(\mathrm{M}-\mathrm{H})^{-} 534.2$, found 534.5 .

\section{2-Benzyl-3-\{[1-(9H-fluoren-9-ylmethoxycarbonylamino)-2-methyl-butyl]-hydroxy-} phosphinoyl\}-propionic acid (3e):
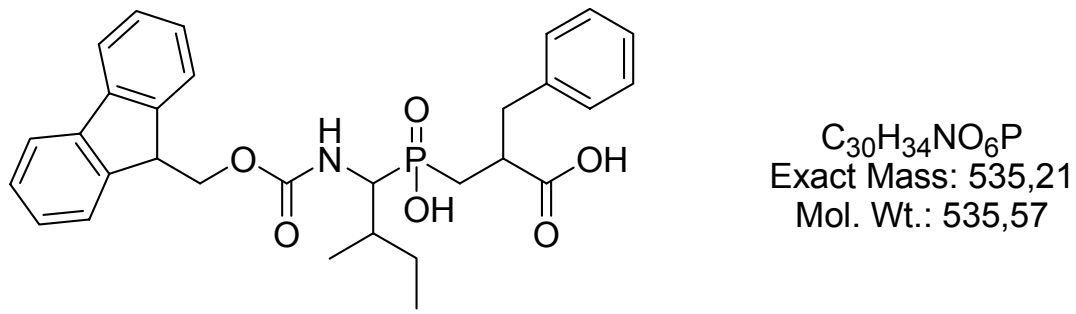

White solid. Yield $61 \%, 332 \mathrm{mg}$. TLC $R_{f}\left(\mathrm{CHCl}_{3} / \mathrm{MeOH} / \mathrm{AcOH}=70 / 5 / 5\right) 0.29$. HPLC $\mathrm{t}_{\mathrm{R}} 44.07 .{ }^{1} \mathrm{H}$ NMR (200 MHz, d 6 -DMSO) $\delta$ 0.82-1.42 (m, 12H), 1.53-2.10 (m, 3H), 2.72-3.10 (m, 3H), 3.453.93, (m, 1H), 4.17-4.36 (m, 3H), 7.17-7.88 (m, 14H); ${ }^{13} \mathrm{C}-\mathrm{NMR}\left(50 \mathrm{MHz}, \mathrm{d}_{6}\right.$-DMSO) $\delta 11.0$, 11.6, 15.7, 16.7, 24.7, 28.7 (d, $\left.{ }^{1} J_{\mathrm{PC}}=91.3 \mathrm{~Hz}\right), 29.2\left(\mathrm{~d},{ }^{1} J_{\mathrm{PC}}=91.3 \mathrm{~Hz}\right), 33.8,34.2,46.7,54.8(\mathrm{~d}$, $\left.{ }^{1} J_{\mathrm{PC}}=97.2 \mathrm{~Hz}\right), 55.3\left(\mathrm{~d},{ }^{1} J_{\mathrm{PC}}=97.2 \mathrm{~Hz}\right), 66.0,120.1,124.8,125.5,126.3,127.1,127.7,128.2$, 129.0, 129.1, 138.9, 140.8, 143.8, 144.0, $156.7\left(\mathrm{~d},{ }^{3} J_{\mathrm{PC}}=5.7 \mathrm{~Hz}\right), 175.4\left(\mathrm{~d},{ }^{3} J_{\mathrm{PC}}=9.9 \mathrm{~Hz}\right) ;{ }^{31} \mathrm{P}-$ NMR (81 MHz, $\mathrm{d}_{6}$-DMSO) $\delta 46.06 / 46.53$. ESMS $m / z$ calcd for $\mathrm{C}_{30} \mathrm{H}_{33} \mathrm{NO}_{6} \mathrm{P}(\mathrm{M}-\mathrm{H})^{-} 534.2$, found 534.4 .

\section{4-[(2-Carboxy-3-phenyl-propyl)-hydroxy-phosphinoyl]-4-(9H-fluoren-9-} ylmethoxycarbonylamino)-butyric acid methyl ester (3f):<smiles>COC(=O)CCC(NC(=O)OCC1c2ccccc2-c2ccccc21)P(=O)(O)CC(Cc1ccccc1)C(=O)O</smiles>

$\mathrm{C}_{30} \mathrm{H}_{32} \mathrm{NO}_{8} \mathrm{P}$ Exact Mass: 565,19 Mol. Wt.: 565,55 
White solid. Yield $60 \%, 340 \mathrm{mg}$. TLC $R_{f}\left(\mathrm{CHCl}_{3} / \mathrm{MeOH} / \mathrm{AcOH}=70 / 5 / 5\right)$ 0.33. HPLC $\mathrm{t}_{\mathrm{R}}$ 38.03/38.47. ${ }^{1} \mathrm{H}$ NMR (200 MHz, $\mathrm{d}_{6}$-DMSO) $\delta 1.62-2.15$ (m, 4H), 2.30-2.45 (m, 2H), 2.78-3.01 (m, 3H), 3.58-3.79 (s+m, 3H+1H), 4.20-4.32 (m, 3H), 7.17-7.91 (m, 14H); ${ }^{13} \mathrm{C}-\mathrm{NMR}(50 \mathrm{MHz}$, $\mathrm{d}_{6}$-DMSO) $\delta 22.9,27.4\left(\mathrm{~d},{ }^{1} J_{\mathrm{PC}}=95.8 \mathrm{~Hz}\right), 27.9\left(\mathrm{~d},{ }^{1} J_{\mathrm{PC}}=95.8 \mathrm{~Hz}\right), 30.1,46.7,49.7\left(\mathrm{~d},{ }^{1} J_{\mathrm{PC}}=105.3\right.$ $\mathrm{Hz}), 50.1\left(\mathrm{~d},{ }^{1} J_{\mathrm{PC}}=105.3 \mathrm{~Hz}\right), 51.4,65.8,120.2,125.4,126.3,127.1,127.7,128.2,129.0,129.1$, $138.8,140.7,143.8,143.9,156.3\left(\mathrm{~d},{ }^{3} J_{\mathrm{PC}}=3.9 \mathrm{~Hz}\right), 172.8,175.2\left(\mathrm{~d},{ }^{3} J_{\mathrm{PC}}=10.1 \mathrm{~Hz}\right) ;{ }^{31} \mathrm{P}-\mathrm{NMR}(81$ MHz, $\mathrm{d}_{6}$-DMSO) $\delta 45.82$. ESMS $m / z$ calcd for $\mathrm{C}_{30} \mathrm{H}_{31} \mathrm{NO}_{8} \mathrm{P}(\mathrm{M}-\mathrm{H})^{-} 564.2$, found 564.5.

\section{2-Benzyl-3-\{[(9H-fluoren-9-ylmethoxycarbonylamino)-phenyl-methyl]-hydroxy- phosphinoyl\}-propionic acid (3g):}
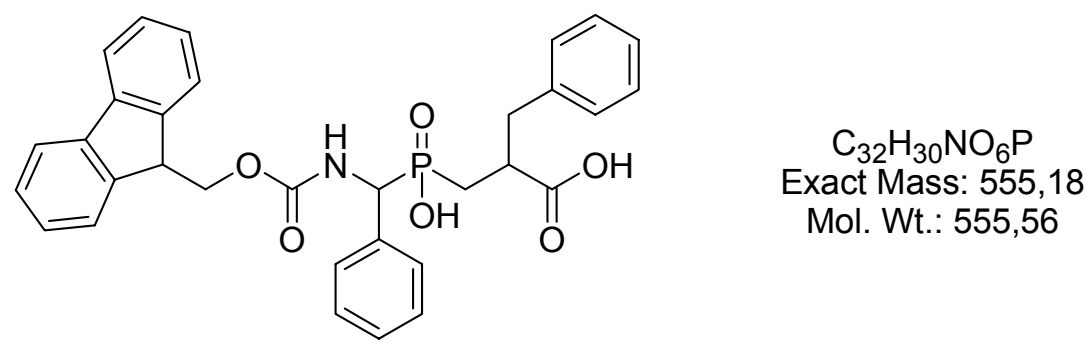

White solid. Yield 73\%, $405 \mathrm{mg}$. TLC $R_{f}\left(\mathrm{CHCl}_{3} / \mathrm{MeOH} / \mathrm{AcOH}=70 / 5 / 5\right) 0.38$. HPLC $\mathrm{t}_{\mathrm{R}} 42.72 .{ }^{1} \mathrm{H}$ NMR (200 MHz, d d $\left.^{-D M S O}\right) \delta 1.64-2.15(\mathrm{~m}, 2 \mathrm{H}), 2.78-2.95$ (m, 3H), 4.17-4.38 (m, 3H), 4.825.08 (m, 1H), 7.13-7.52 (m, 14H), 7.75-7.91 (m, 4H), 8.37-8.52 (m, 1H); ${ }^{13} \mathrm{C}-\mathrm{NMR}\left(50 \mathrm{MHz}, \mathrm{d}_{6}-\right.$ DMSO) $\delta 27.7\left(\mathrm{~d},{ }^{1} J_{\mathrm{PC}}=91.9 \mathrm{~Hz}\right), 28.2\left(\mathrm{~d},{ }^{1} J_{\mathrm{PC}}=91.9 \mathrm{~Hz}\right), 46.6,55.3\left(\mathrm{~d},{ }^{1} J_{\mathrm{PC}}=97.7 \mathrm{~Hz}\right), 55.8(\mathrm{~d}$, $\left.{ }^{1} J_{\mathrm{PC}}=97.7 \mathrm{~Hz}\right), 66.2,120.1,125.4,125.5,126.3,127.1,127.7,128.0,128.2,129.0,129.1,136.6$, $138.8,140.7,143.7,143.9,156.2\left(\mathrm{~d},{ }^{3} J_{\mathrm{PC}}=4.5 \mathrm{~Hz}\right), 175.3\left(\mathrm{~d},{ }^{3} J_{\mathrm{PC}}=9.3 \mathrm{~Hz}\right) ;{ }^{31} \mathrm{P}-\mathrm{NMR}(81 \mathrm{MHz}$, $\mathrm{d}_{6}$-DMSO) $\delta 41.71$. ESMS $m / z$ calcd for $\mathrm{C}_{32} \mathrm{H}_{29} \mathrm{NO}_{6} \mathrm{P}(\mathrm{M}-\mathrm{H})^{-} 554.2$, found 554.4. 
2-Benzyl-3-\{[2-benzyloxy-1-(9H-fluoren-9-ylmethoxycarbonylamino)-ethyl]-hydroxyphosphinoyl\}-propionic acid (3h):
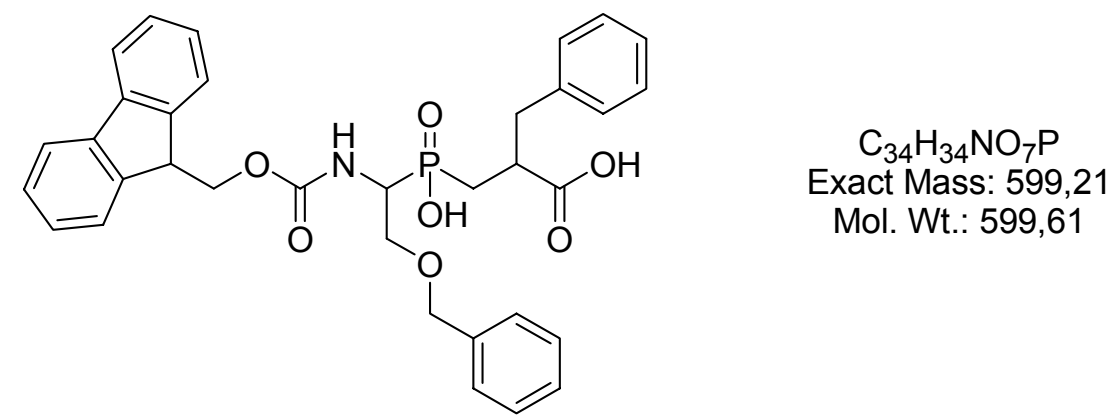

White solid. Yield 67\%, $402 \mathrm{mg}$. TLC $R_{f}\left(\mathrm{CHCl}_{3} / \mathrm{MeOH} / \mathrm{AcOH}=70 / 5 / 5\right)$ 0.30. HPLC $\mathrm{t}_{\mathrm{R}} 45.99 .{ }^{1} \mathrm{H}$ NMR (200 MHz, d d $\left.^{-D M S O}\right) \delta 1.65-2.15(\mathrm{~m}, 2 \mathrm{H}), 2.79-3.02(\mathrm{~m}, 3 \mathrm{H}), 3.62-3.81(\mathrm{~m}, 2 \mathrm{H}), 3.99-$ $4.18(\mathrm{~m}, 1 \mathrm{H}), 4.19-4.35$ (m, 3H), 4.48 (s, 2H), 7.17-7.45 (m, 14H), 7.66-7.91 (m, 5H); ${ }^{13} \mathrm{C}-\mathrm{NMR}$ (50 MHz, $\mathrm{d}_{6}$-DMSO) $\delta 28.3\left(\mathrm{~d},{ }^{1} J_{\mathrm{PC}}=90.3 \mathrm{~Hz}\right), 28.7\left(\mathrm{~d},{ }^{1} J_{\mathrm{PC}}=90.3 \mathrm{~Hz}\right), 46.7,51.3\left(\mathrm{~d},{ }^{1} J_{\mathrm{PC}}=100.8\right.$ $\mathrm{Hz}), 51.7\left(\mathrm{~d},{ }^{1} J_{\mathrm{PC}}=100.8 \mathrm{~Hz}\right), 66.0,67.5,71.9,120.1,125.4,126.3,127.1,127.4,127.5,127.7$, 128.2, 129.0, 136.6, 138.2, 138.8, 140.7, 143.9, $156.4\left(\mathrm{~d},{ }^{3} J_{\mathrm{PC}}=5.1 \mathrm{~Hz}\right), 175.2\left(\mathrm{~d},{ }^{3} J_{\mathrm{PC}}=7.3 \mathrm{~Hz}\right)$; ${ }^{31} \mathrm{P}-\mathrm{NMR}\left(81 \mathrm{MHz}, \mathrm{d}_{6}\right.$-DMSO) $\delta 44.19 / 44.27$. ESMS $m / z$ calcd for $\mathrm{C}_{34} \mathrm{H}_{33} \mathrm{NO}_{7} \mathrm{P}(\mathrm{M}-\mathrm{H})^{-}$598.2, found 598.4.

\section{2-Benzyl-3-\{[(9H-fluoren-9-ylmethoxycarbonylamino)-(1H-imidazol-4-yl)-methyl]-hydroxy-} phosphinoyl\}-propionic acid (3i):
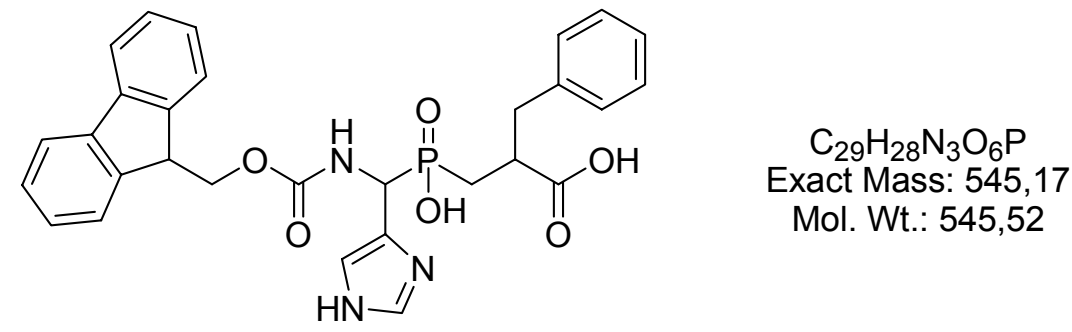

White solid. Yield 69\%, $377 \mathrm{mg}$. TLC $R_{f}\left(\mathrm{CHCl}_{3} / \mathrm{MeOH} / \mathrm{AcOH}=7 / 2 / 1\right) \quad 0.32$. HPLC $t_{\mathrm{R}}$ 27.86/28.01. ${ }^{1} \mathrm{H}$ NMR (200 MHz, d d $\left.^{-D M S O}\right) \delta 1.62-2.10(\mathrm{~m}, 2 \mathrm{H}), 2.78-3.19(\mathrm{~m}, 3 \mathrm{H}), 4.05-4.31$ $(\mathrm{m}, 3 \mathrm{H}), 4.78-5.10(\mathrm{~m}, 1 \mathrm{H})$ 7.15-8.21 (m, 16H) 8.45-8.80 (m, 1H); ${ }^{13} \mathrm{C}-\mathrm{NMR}\left(50 \mathrm{MHz}, \mathrm{d}_{6^{-}}\right.$ 
DMSO) $\delta 30.0\left(\mathrm{~d},{ }^{1} J_{\mathrm{PC}}=92.2 \mathrm{~Hz}\right), 47.2,48.2\left(\mathrm{~d},{ }^{1} J_{\mathrm{PC}}=97.6 \mathrm{~Hz}\right), 49.1\left(\mathrm{~d},{ }^{1} J_{\mathrm{PC}}=97.6 \mathrm{~Hz}\right), 66.9$, 117.1, 120.8, 126.6, 127.8, 128.3, 128.7, 129.7, 131.9, 134.2, 140.1, 141.3, 144.3, 144.5, 156.7 $\left(\mathrm{d},{ }^{3} J_{\mathrm{PC}}=5.8 \mathrm{~Hz}\right), 176.4\left(\mathrm{~d},{ }^{3} J_{\mathrm{PC}}=7.2 \mathrm{~Hz}\right) ;{ }^{31} \mathrm{P}-\mathrm{NMR}\left(81 \mathrm{MHz}, \mathrm{d}_{6}\right.$-DMSO) $\delta 31.87$. ESMS $\mathrm{m} / \mathrm{z}$ calcd for $\mathrm{C}_{29} \mathrm{H}_{27} \mathrm{~N}_{3} \mathrm{O}_{6} \mathrm{P}(\mathrm{M}-\mathrm{H})^{-} 544.2$, found 544.4.

\section{2-\{[1-(9H-Fluoren-9-ylmethoxycarbonylamino)-ethyl]-hydroxy-phosphinoylmethyl\}-4-} methyl-pentanoic acid (3j):

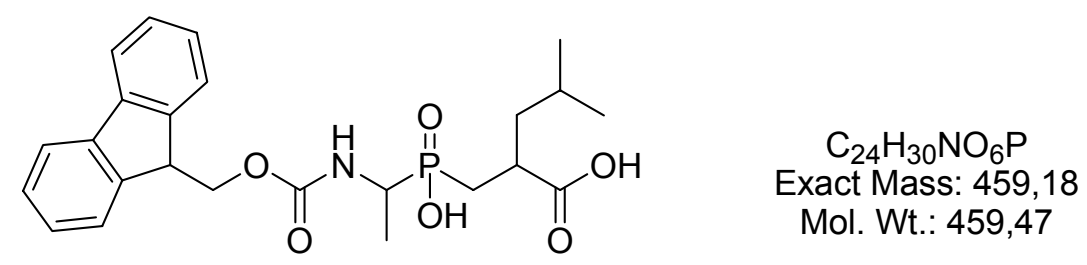

White solid. Yield 58\%, $281 \mathrm{mg}$. TLC $R_{f}\left(\mathrm{CHCl}_{3} / \mathrm{MeOH} / \mathrm{AcOH}=70 / 5 / 5\right) 0.25$. HPLC $\mathrm{t}_{\mathrm{R}} 37.32 .{ }^{1} \mathrm{H}$ NMR (200 MHz, d $\mathrm{d}_{6}$-DMSO) $\delta 1.05-1.75(\mathrm{~m}, 13 \mathrm{H}), 1.82-2.10(\mathrm{~m}, 1 \mathrm{H}), 2.58-2.80(\mathrm{~m}, 1 \mathrm{H}), 3.65-$ $3.90(\mathrm{~m}, 1 \mathrm{H}), 4.15-4.39(\mathrm{~m}, 3 \mathrm{H}), 7.21-7.90(\mathrm{~m}, 9 \mathrm{H}) ;{ }^{13} \mathrm{C}-\mathrm{NMR}\left(50 \mathrm{MHz}, \mathrm{d}_{6}\right.$-DMSO) $\delta 21.8,23.0$, 25.6, 28.6 (d, $\left.{ }^{1} J_{\mathrm{PC}}=89.6 \mathrm{~Hz}\right), 42.7,45.8\left(\mathrm{~d},{ }^{1} J_{\mathrm{PC}}=106.3 \mathrm{~Hz}\right), 46.2\left(\mathrm{~d},{ }^{1} J_{\mathrm{PC}}=106.3 \mathrm{~Hz}\right), 47.3,65.9$, $120.2,125.3,127.1,127.7,140.8,143.9,155.9\left(\mathrm{~d},{ }^{3} J_{\mathrm{PC}}=5.5 \mathrm{~Hz}\right), 176.4\left(\mathrm{~d},{ }^{3} J_{\mathrm{PC}}=7.5 \mathrm{~Hz}\right) ;{ }^{31} \mathrm{P}-$ NMR (81 MHz, $\left.\mathrm{d}_{6}-\mathrm{DMSO}\right) \delta 46.78 / 47.01$. ESMS $m / z$ calcd for $\mathrm{C}_{24} \mathrm{H}_{29} \mathrm{NO}_{6} \mathrm{P}(\mathrm{M}-\mathrm{H})^{-} 458.2$, found 458.3 .

3-\{[(9H-Fluoren-9-ylmethoxycarbonylamino)-phenyl-methyl]-hydroxy-phosphinoyl\}-2methyl-propionic acid (3k):<smiles>CC(CP(=O)(O)C(NC(=O)OCC1c2ccccc2-c2ccccc21)c1ccccc1)C(=O)O</smiles> 
White solid. Yield 54\%, $259 \mathrm{mg}$. TLC $R_{f}\left(\mathrm{CHCl}_{3} / \mathrm{MeOH} / \mathrm{AcOH}=70 / 5 / 5\right)$ 0.15. HPLC $\mathrm{t}_{\mathrm{R}} 34.56 .{ }^{1} \mathrm{H}$

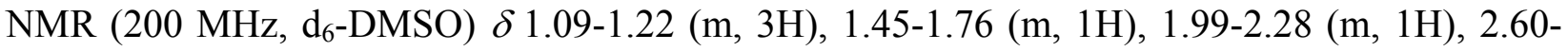
$2.78(\mathrm{~m}, 1 \mathrm{H}), 4.05-4.35(\mathrm{~m}, 3 \mathrm{H}), 4.80-5.05$ (m, 1H), 7.20-7.46 (m, 7H), 7.76-7.90 (m, 5H), 8.25$8.35(\mathrm{~m}, 2 \mathrm{H}) ;{ }^{13} \mathrm{C}-\mathrm{NMR}\left(50 \mathrm{MHz}, \mathrm{d}_{6}\right.$-DMSO) $\delta 27.7,29.7\left(\mathrm{~d},{ }^{1} J_{\mathrm{PC}}=89.8 \mathrm{~Hz}\right), 33.3,46.7,55.8(\mathrm{~d}$, $\left.{ }^{1} J_{\mathrm{PC}}=98.6 \mathrm{~Hz}\right), 66.2,120.2,125.4,125.6,127.1,127.7,128.1,136.5,140.8,143.8,143.9,156.3$ $\left(\mathrm{d},{ }^{3} J_{\mathrm{PC}}=8.8 \mathrm{~Hz}\right), 176.7\left(\mathrm{~d},{ }^{3} J_{\mathrm{PC}}=12.1 \mathrm{~Hz}\right) ;{ }^{31} \mathrm{P}-\mathrm{NMR}\left(81 \mathrm{MHz}, \mathrm{d}_{6}-\mathrm{DMSO}\right) \delta 42.77$. ESMS $m / z$ calcd for $\mathrm{C}_{26} \mathrm{H}_{25} \mathrm{NO}_{6} \mathrm{P}(\mathrm{M}-\mathrm{H})^{-} 478.1$, found 478.3 .

\section{3-\{[1-(9H-Fluoren-9-ylmethoxycarbonylamino)-3-methyl-butyl]-hydroxy-phosphinoyl $\}-$}

\section{propionic acid (31):}
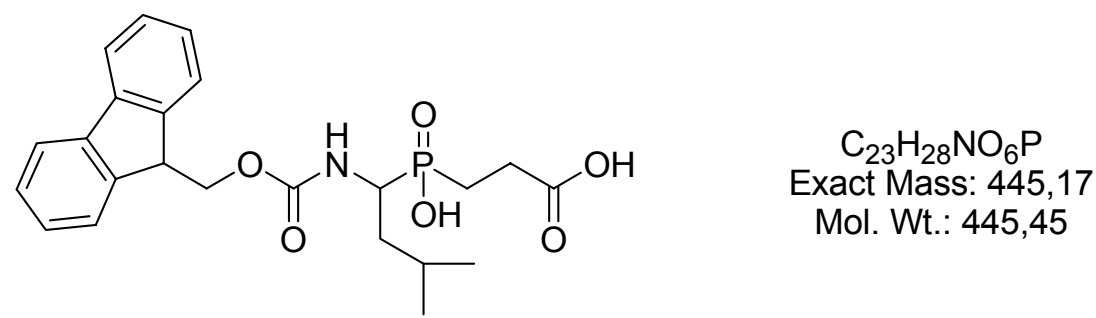

White solid. Yield 42\%, $187 \mathrm{mg}$. TLC $R_{f}\left(\mathrm{CHCl}_{3} / \mathrm{MeOH} / \mathrm{AcOH}=7 / 2 / 1\right)$ 0.29. HPLC $\mathrm{t}_{\mathrm{R}} 33.90 .{ }^{1} \mathrm{H}$ NMR (200 MHz, d6-DMSO) $\delta$ 0.79-0.91 (m, 6H), 1.35-1.86 (m, 5H), 1.91-2.51 (m, 2H), 3.55$3.82(\mathrm{~m}, 1 \mathrm{H}), 4.17-4.35(\mathrm{~m}, 3 \mathrm{H}), 7.26-7.44(\mathrm{~m}, 4 \mathrm{H}), 7.57-7.73(\mathrm{~m}, 3 \mathrm{H}), 7.86-7.90(\mathrm{~m}, 2 \mathrm{H}) ;{ }^{13} \mathrm{C}-$ NMR (50 MHz, d6-DMSO) $\delta$ 20.7, 20.9, 21.0, 22.5, 23.4, 23.7, 24.1, 26.4, 35.7, 46.8, 48.1 (d, $\left.{ }^{1} J_{\mathrm{PC}}=108.9 \mathrm{~Hz}\right), 65.6,120.1,125.2,125.3,127.1,127.6,140.7,143.7,143.9,156.2\left(\mathrm{~d},{ }^{3} J_{\mathrm{PC}}=4.6\right.$ $\mathrm{Hz}), 173.7\left(\mathrm{~d},{ }^{3} J_{\mathrm{PC}}=16.5 \mathrm{~Hz}\right)$; ${ }^{31} \mathrm{P}-\mathrm{NMR}\left(81 \mathrm{MHz}, \mathrm{d}_{6}\right.$-DMSO) $\delta 47.45$. ESMS $\mathrm{m} / \mathrm{z}$ calcd for $\mathrm{C}_{23} \mathrm{H}_{27} \mathrm{NO}_{6} \mathrm{P}(\mathrm{M}-\mathrm{H})^{-}$444.2, found 444.3.

Solid Phase Synthesis of compound 4: Synthesis was carried out on pins (SynPhase Lanterns, L-series pins, loading: $15 \mu \mathrm{mol}$ per pin, Mimotopes, France). Fmoc deprotection: Fmoc-Rink amide derivatized pins were treated with a $20 \%$ piperidine/DMF solution $(1 \mathrm{~mL} / \mathrm{pin}, 2 \times 30 \mathrm{~min}$, rt). Each time, the solvent was decanted, and the pins were washed with DMF $(2 \times 5$ min, 1 mL/pin), DCM (2x5 min, $1 \mathrm{~mL} /$ pin) and air-dried. Fmoc-Tryptophan coupling: The deprotected pins were treated with a preactivated $(10 \mathrm{~min})$ mixture of Fmoc-tryptophan ( $3 \mathrm{eq}$ in $1 \mathrm{~mL} / \mathrm{pin})$, DIC (3 eq) and HOBt (3 eq) in a DCM/DMF (1:1) solution for 4-5 h, rt. Then, the solution was 
decanted and the pins were washed with DCM (2x5 min, $1 \mathrm{~mL} / \mathrm{pin}), \mathrm{DMF}(2 \times 5 \mathrm{~min}, 1 \mathrm{~mL} / \mathrm{pin})$, and DCM (2x5 min, $1 \mathrm{~mL} /$ pin) and briefly air-dried. Negative Kaiser test. Fmoc-deprotection was repeated as described above. Phosphinic block coupling: A solution of the dipeptidic block 3d (1.1 eq/pin) was added to pins in dry DCM (0.25 mL/pin) containing DIPEA (1.9 eq), HOBt (1 eq) and $\mathrm{EDCHCl}(4 \mathrm{eq})$. The reaction mixture was shaken from time to time for $5 \mathrm{~h}$. The coupling was stopped by decanting the solution. The pins were washed with DCM $(2 \times 5 \mathrm{~min}, 1$ $\mathrm{mL} / \mathrm{pin}), \operatorname{DMF}(2 \times 5 \mathrm{~min}, 1 \mathrm{~mL} / \mathrm{pin})$, and DCM (2x5 min, $1 \mathrm{~mL} / \mathrm{pin})$ and briefly air-dried. Coupling step was repeated once more when Kaiser test was negative. After removal of Fmocgroup and washings, coupling with $3 \mathrm{eq} / \mathrm{pin}$ of FmocGlu( $\left.\mathrm{OBu}^{\mathrm{t}}\right) \mathrm{OPfp}$ using HOBt (3 eq) in 1 DMF mL/pin, until a negative Kaiser test. Fmoc-deprotection was repeated as described above. Acetylation step was performed using 6 eq of $\mathrm{AcOH}$, DIC and $\mathrm{HOBt}$ in DMF $(1 \mathrm{~mL} / \mathrm{pin})$ for $3 \mathrm{~h}$ at rt. Then, the solution was decanted and the pins were washed with DCM ( $\times 5 \mathrm{~min}, 1 \mathrm{~mL} / \mathrm{pin})$, DMF (2x5 min, $1 \mathrm{~mL} / \mathrm{pin})$, and DCM (2x5 min, $1 \mathrm{~mL} / \mathrm{pin})$ and briefly air-dried. Negative Kaiser test. Cleavage from pins was achieved using TFA/TIS/ $\mathrm{H}_{2} \mathrm{O}$ 95:2.5:2.5 (1 mL/pin). The residue was concentrated in vacuo and precipitated with dry $\mathrm{Et}_{2} \mathrm{O}$.

\section{4-Acetylamino-4-[1-(\{2-[1-carbamoyl-2-(1H-indol-3-yl)-ethylcarbamoyl]-3-phenyl-propyl\}-} hydroxy-phosphinoyl)-3-methyl-butylcarbamoyl]-butyric acid (4):
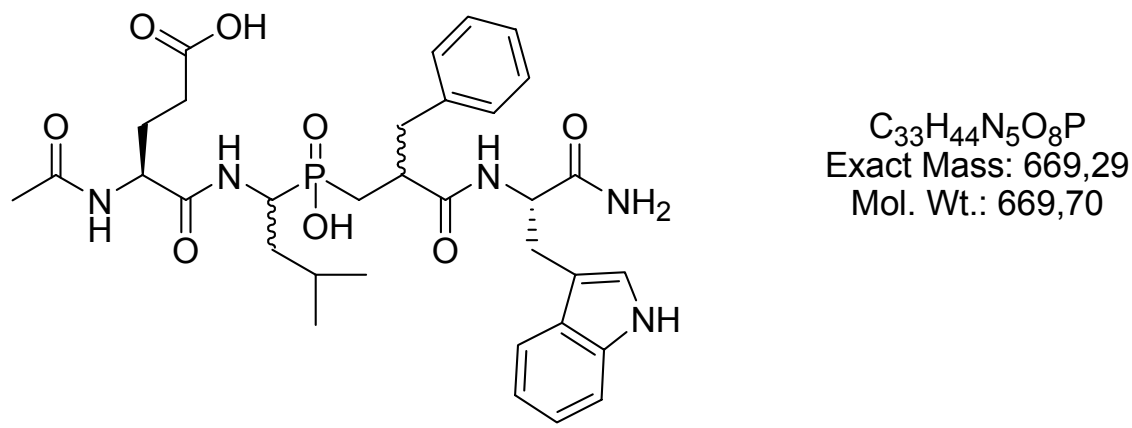

White solid. Yield 85\%, $30 \mathrm{mg}$. 3 pins were used, $50 \mathrm{mg}$ of 3d block for 2 couplings, $30 \mathrm{mg}$ of 4 obtained, containing the 4 diasteroisomers in $89 \%$ purity, as shown by HPLC. TLC $R_{f}\left(\mathrm{CHCl}_{3} / \mathrm{MeOH} / \mathrm{AcOH}=7 / 2 / 1\right)$ 0.12. HPLC $\mathrm{t}_{\mathrm{R}} 21.01 / 21.76 / 29.12 / 36.44$. ESMS $\mathrm{m} / z$ calcd for $\mathrm{C}_{33} \mathrm{H}_{43} \mathrm{~N}_{5} \mathrm{O}_{8} \mathrm{P}(\mathrm{M}-\mathrm{H})^{-} 668.3$, found 668.4. 